\title{
A organização do abastecimento do hospital público a partir da cadeia produtiva: uma abordagem logística para a área de saúde
}

\author{
Production chain supply management for public hospitals: \\ a logistical approach to healthcare
}

M aria Infante ${ }^{1}$

M aria Angélica Borges dos Santos ${ }^{1,2}$

${ }^{1} \mathrm{~N}$ úcleo de Tecnologiaem

Abstract Despite their importance for hospital operations, discussions of healthcare organization logistics and supply and materials management are notably lacking in Brazilian literature. This paper describes a methodology for organizing the supply of medical materials in publichospitals, based on an action-research approach. Interventions were based on the assumption that a significant portion of problems in Brazil's National $\mathrm{H}$ ealth System (SUS) facilities derive from the fact that their clinical and administrative departments do not see themselves as bel onging to the same production chain - neither the hospital nor the supply department is aware of what the other produces. The development of the methodology and its main steps are presented and discussed, against a background of recent literature and total quality and supply chain management concepts.

Key words Hospital management, Hospital materials management, Supply chain management, Total quality management
Resumo Apesar de sua relevância para a operação dos hospitais, a discussão sobre processo logístico, abastecimento e cadeia de suprimentos em organizações de saúde está notadamente ausente da literatura nacional. 0 presente artigo descreve uma metodologia para a organização do setor de abastecimento de materiais médico-hospitalares de hospitais públicos, desenvolvida com base em uma abordagem de pesquisa-ação. A premissa que norteou as intervenções propostas para o setor foi a de que grande parte dos problemas em unidades médico-assistenciais do Sistema Ú ni co de Saúde nessa área decorria do fato de setores clínicos e administrativos não se identificarem como uma cadeia produtiva integrada - 0 que os setores clínicos produzem não é transparente para os setores ligados ao abastecimento e vice-versa. São apresentados um histórico do de senvolvimento da metodologia, a descrição desuas princi pais etapas e uma discussão à luz da literatura recente e de alguns conceitos de qualidade e de gestão da cadeia de suprimentos.

Palavras-chave Administração hospitalar, Administração de materiais no hospital, Gestão da cadeia desuprimentos, Gestão da QualidadeTotal 
M ateriais e logística são, juntamente com recursos humanos e administração financeira, fatores críticos para o desenvolvimento de atividades de atenção à saúde e para a excelência operacional da organização hospitalar ${ }^{1}$. Entretanto, embora a irregularidade do abastecimento e a "falta de material" sejam problemas freqüentes em serviços públicos de saúde e tenham significativos impactos negativos sobre seus desempenhos e imagem junto aos profissionais eà população, a discussão sobre processo logístico, abastecimento e cadeia de suprimentos ${ }^{2}$ está notadamente ausente da literatura nacional.

A tendência no setor público parece ser reduzir todos os problemas deabastecimento dos serviços de saúde à insuficiência de recursos orçamentários. É bem verdade que os efeitos deletérios dos períodos de desabastecimento provocados por disfunções da execução orçamentária não são desprezíveis. Entretanto, também são notórios os desperdícios e a má utilização de insumos e equipamentos, a escassa qualificação dos profissionais da área de abastecimento ea pouca atenção ao planejamento logístico nas organizações públicas de saúde.

Uma organização de saúde éum sistema produtivo de atenção à saúde, onde o setor de abastecimento integra-se como subsistema para atender asnecessidades de insumos (materiais de consumo) e de equipamentos (materiais permanentes) daqueles que desenvolvem e disponibilizam os produtos, que são os profissionais de saúde.

As atividades de atenção à saúde são atividades complexas, assentadas sobre uma cadeia produtiva que incorpora seqüências de ações definidas para a geração de seus produtos (os chamados "procedimentos"). Cada procedimento demanda um mix específico de insumos (bens) e processos de trabalho (serviços), cuja composição pode variar entre diferentes organizações e atésegundo os diferentestipos de pacientes eprofissionais de uma mesma organização.

Não só os produtos oferecidos em organizações de saúde são complexos e pressupõem elevada qualificação profissional, mas os insumos utilizados em sua produção são cada vez mais sofisticados e numerosos. Vecina $\&$ Reinhardt ${ }^{3}$ estimam que o sistema de materiais de um hospital comporte entre 3.000 a 6.000 itens regularmente adquiridos, na dependência do perfil das atividades desenvolvidas pela organização. Esses itens incluem os chamados materiais de consumo médico-hospitalares, medicamentos e reagentes para testes diagnósticos, além de mate- riais especiais, como órteses e próteses, e itens gerais de escritório, limpeza e alimentos.

Dados sobre a participação dos materiais hospitalares (inclusive medicamentos) no orçamento dos hospitais no Reino Unido indicam que ela pode corresponder de 13 a $17 \%$ dos custos hospitalares, não diferindo substancialmentedas estimativas nacionais, de $15 \%$ a $25 \%$ 3,4. Inúmeros trabalhos apontam a economia potencial e os ganhos de qualidade na assistência advindos da racionalização do sistema de abastecimento, destacando a importância de incorporar os conceitos de logística e cadeia de suprimentos para organizar o sistema produtivo do hospital ${ }^{15,6,7,7}$.

$\mathrm{Na}$ visão ampliada da cadeia de suprimentos, uma cadeia produtiva abrange desde o desenvolvimento de um produto (que, nas atividades de atenção à saúde, corresponderia à introdução de um novo processo de trabalho e seus insumos correspondentes), passando pelo fornecedor de insumos, até a efetiva oferta do produto ao mercado consumidor. 0 conceito de cadeia de suprimentos destaca a integração interna eexterna dos participantes de todas as etapas - "desenvolvedores" do produto, fornecedores de insumos, responsáveis pela logística de aquisição, armazenamento e distribuição dos insumos e responsáveis pela fabricação e distribuição final do produto, incluindo o próprio clientefinal².

0 presente artigo descreve uma metodologia para a otimização do setor de abastecimento hospitalar público - aqui conceituado como o de gestão demateriais médico-hospitalares- desenvolvida no Núcleo de Logística e Tecnologia em Saúde (NUTEC) da Escola Nacional de Saúde Pública (ENSP), com base em técnicas de pesquisa-ação. São apresentados um histórico do desenvolvimento da metodologia, a descrição de suas principais etapas e uma discussão sobre 0 tema e alguns conceitos de qualidade e gestão da cadeia de suprimentos à luz da literatura internacional recente.

\section{Materiais emétodos}

A otimização do abastecimento hospitalar a partir da visão da cadeia produtiva é uma metodologia em evolução, desenvolvida sob a égide dos princípios da melhoria contínua da qualidade e do modelo de pesquisa-ação. A pesquisa-ação é uma abordagem metodológica que implica um significativo afastamento do modelo tradicional da pesquisa acadêmica, sendo usada para estu- 
dar soluções para problemas originados na sociedade e nas organizações.

$\mathrm{Na}$ pesquisa-ação, programas de melhoria organizacional são encarados como situações de pesquisa e constituem bases para o avanço de teorias e o desenvolvimento de práticas. Os problemas escolhidos como objeto de pesquisa e as próprias pesquisas são desenvolvidos mais na perspectiva de busca de soluções, que compreendem prescrições e ações, do quecom vistas à descrição e à análise, abordagem privilegiada pelo modelo acadêmico tradicional ${ }^{8}$. Em termos metodológicos, o pesquisador não atua como observador independente, mas torna-se um participante na busca por soluções, atuando como facilitador da ação e reflexão e elaborando um arcabouço teórico e de intervenções que evolui à medida que os dados vão sendo colhidos e as idéias desenvolvidas. A técnica de intervenção é adaptada à medida que vai sendo usada, e a cada aplicação aumenta a compreensão sobre seu escopo e suas limitações 9 ,10.

A presente pesquisa começou a ser esboçada no início da década de 90, a partir do interesse dos profissionais do NUTEC por questões cotidianas de operações em organizações de saúde em função desuas vivências anteriores como gestores, bem como da leitura dos manuais da série M EDEX, da Faculdade de M edicina da Universidade do Havaí ${ }^{11}$. Esses manuais apresentavam uma abordagem para aperfeiçoar sistemas gerenciais (financeiro, recursos humanos, materiais, de transporte e informações) em organizações de saúde, que destacava a importância de integrar, em oficinas ou grupos de discussão, representantes do chamado sistema produtivo (áreasfim) edos sistemas gerenciais (áreas meio), além de responsáveis pela elaboração de políticas e pela sua implementação. Com isso, aumentariam a legitimidadee a efetivi dade das soluções para problemas comuns do gerenciamento de organizações de saúde.

Através da aplicação dessa premissa, foi desenvolvida uma abordagem do tipo pesquisaação ao estudo de sistemas gerenciais de vários hospitais e secretarias municipais do estado do Rio de Janeiro, que incluíram oficinas para reestruturação dos setores administrativos de dois hospitais federais especializados, entre 1991 e 1993, e projetos de implantação de Núcleos de Q ualidade em Saúde em uma secretaria municipal de saúde e em um Hospital geral no Estado do Rio de Janeiro, em 1996.
A partir de 1998, o sistema de abastecimento foi sendo definido como o foco primordial de interesse do grupo. As sucessivas oficinas realizadas sobre o tema ao logo da década de 90 de monstravam que era difícil abastecer com racionalidade uma unidade de saúde na visão exclusiva das prateleiras dos almoxarifados, levandose em conta somente alguns indicadores como estoquemínimo, estoque máximo ou estoque de risco. Era preciso desenhar uma abordagem que contemplasse as especificidades das organizações de saúde.

Técnicas de problematização e outras ferramentas da gestão da qualidade foram sendo aos poucos incorporadas às oficinas de trabal ho $0^{12,13}$ e, com o tempo, foi sendo esboçada a visão da organização como uma cadeia de atividades, em que os sucessivos elos da cadeia comportam-se como clientes e fornecedores, respectivamente, dos elos precedentes e seguintes da cadeia. Assim, uma organização passava a ser concebida como uma cadeia de "clientes" e "fornecedores", que precisam estabelecer relacionamentos delongo prazo para atingir um objetivo comum.

Em 1998, foram realizados dois projetos (o primeiro em um hospital especializado e outro em um hospital geral) na área de organização de informações e desenvolvimento gerencial para a área de logística eabastecimento, que contribuíram para dar corpo à metodologia aqui apresentada em seu formato original.

Em 1999, a metodologia para a organização do abastecimento hospitalar desenvolvida a partir desses trabalhos foi aplicada, pela primeira vez, em um hospital público federal no Rio de Janeiro. A lógica da metodologia já incorporava não apenas os princípios da qualidade, mas também, de modo incipiente, o conceito de cadeia produtiva.

Desde então, trabalhos de otimização do sistema de abastecimento já foram executados em outros três hospitais públicos de vários portes e especificidades, sendo um hospital geral de grande porte (2001-2), um especializado em doenças cardíacas (2002-3) e um hospital universitário (2005-6). A demanda pela aplicação da metodologia em geral parte da própria direção dos hospitais, que entra em contato com a mesma durante cursos realizados por seu corpo clínico e gerencial naENSP.

Os resultados apresentados sintetizam as bases e a evolução da metodologia em seus diferentes locais de aplicação. 
Resultados

As intervenções propostas para o setor de abaste cimento hospitalar foram norteadas pela premissa de que grande parte dos problemas em unidades médico-assistenciais do Sistema Único de Saúde nessa área decorria do fato de setores clínicos e administrativos não se identificarem como uma cadeia produtiva integrada. Ou seja, o que o hospital produzia não era transparente para o sistema deabastecimento evice-versa. Adicionalmente, não havia um sistema próprio de informações para 0 abastecimento que correlacionasse produtos e insumos, reconhecendo os processos de trabalho do sistema produtivo e valorizando as informações dos profissionais de saúde.

$\mathrm{Na}$ cadeia produtiva hospitalar, os produtos eram "desenvolvidos" e "aplicados" pelos profissionais de saúde e, portanto, precisavam ser racionalmente customizados aos processos de trabal ho dosmesmos. M odificações de procedimentos e processos de trabalho nas organizações de saúde, com a inclusão de novos profissionais, técnicas, equipamentos einsumos, bem como as alterações desencadeadas no plano logístico hospitalar por mudanças do meio externo ou do meio interno, tornavam obrigatória a participação dos médicos, enfermeiros etécnicos de saúde no planejamento do sistema de abastecimento, como fonte permanente de informações.

O sistema de abastecimento era concebido como composto por quatro subsistemas: planejamento, controle, guarda e distribuição e compras. 0 subsistema de planejamento estaria ligado às tarefas de seleção, especificação e incorporação de novos materiais e sobre ele incidiam as primeiras intervenções preconizadas na metodologia. 0 subsistema de controle era o responsável por acompanhar e valorar estoques. Ao subsistema de guarda e distribuição cabia receber, conferir, estocar de forma organizada e distribuir internamente os materiais, enquanto o subsistema de compras era o responsável pela aquisição de materiais. A equipe do subsistema de compras deveria ter um bom conhecimento do mercado de materiais, cabendo-Ihe organizar 0 cadastro de fornecedores, elaborar editais e implantar as formas mais eficientes disponíveis de adquirir materiais.

Partindo-se dessa segmentação, a organização do sistema de abastecimento e da logística hospitalar a partir do sistema produtivo era desenvolvida em três etapas: (i) implantação ou redirecionamento do subsistema de planejamento, (ii) reorganização dos subsistemas de guarda e distribuição e de compras e (iii) estabelecimento da distribuição interna automática por entrepostagem.

\section{Primeira etapa}

A primeira etapa consistia em implantar ou redirecionar o subsistema de planejamento, cuja principal função passava a ser estabelecer uma ponte com o restante da cadeia produtiva, definindo uma linguagem comum. As pesquisas mostravam que, de um modo geral, esse subsistema atuava de modo burocrático, mantinha escassa interação com a área clínica e precisava ter sua dinâmica de funcionamento redirecionada para contemplar as especificidades da área de saúde.

O trabalho iniciava-se pela criação de círculos de qualidade ou grupos locais, estabelecendo-se um primeiro intercâmbio entre as equipes do abastecimento e dos setores clínicos. Como estratégia para definir as novas bases de relacionamento, eram utilizadas técnicas de problematização que, por se prestarem a promover uma aproximação e a troca de informações entre distintos segmentos da organização, são empregadas para implantar os princípios da qualidade em empresas ${ }^{14}$. Cada encontro entre os profissionais das distintas áreas pretendia ser um momento de esclarecimento e aprendizado, contribuindo para criar um clima de confiança mútua e de co-responsabilização.

Esses grupos locais constituiriam a base para a oportuna criação de Comissão Permanente de Padronização de M aterial M édico-H ospitalar, que, com a consolidação da reorganização do abastecimento, passaria a ser uma instância permanente de gerenciamento técnico do abastecimento na organização. Entre as suas atribuições formais estariam incluídos gerenciar as atualizações da grade de materiais, organizar pareceres técnicos sobre materiais e construir indicadores dequalidadee produtividade, estabelecendo uma troca permanente de informações entre 0 abastecimento e a clínica.

A primeira ferramenta utilizada para implementar o planejamento a partir da nova visão era a pesquisa de insumos médico-hospitalares, que objetivava o mapeamento de todos os procedimentos executados pelas várias equipes de profissionais de saúde em cada setor hospitalar, a especificação de todos os insumos necessários para a produção de cada procedimento, bem como a quantificação de sua produção mensal. A seleção dos insumos necessários ao funciona- 
mento do hospital ia se constituindo a partir deste momento de identificação.

A pesquisa de insumos compreendia quatro fases: a fase do levantamento; a fase do check-list (organização da denominação dos procedimentos e insumos correspondentes por setor); a fase de sistematização (em que era feita a organização dos insumos para o conjunto do hospital, com vistas a padronizar as especificações para insumos comuns a vários setores); e a fase de validação (em que a nova listagem voltava a cada setor para ser confirmada).

Asinformações fornecidas pelos profissionais de saúde de cada setor permitiam a construção de "matrizes insumo-produto", que passavam a compor um banco de dados para organizar o abastecimento. Asmatrizes estabeleciam uma correlação significativa entre uso de insumos e procedimentos clínico-cirúrgicos efetuados por médicos, enfermagem e outros profissionais de saúde e uma padronização do tipo edas quantidades de insumo utilizados em cada procedimento. A informática era um auxiliar indispensável nessa etapa, pois permitia a criação dos bancos de dados de produtores, produtos e insumos, além da elaboração de programas e relatórios.

Integrava também esta etapa um levantamento de insumos existentes no almoxarifado do hospital, produzido pela área de guarda e distribuição. Esses dados permitiam comparar o que se guardava e distribuía no hospital antes da realização da pesquisa e o que se passava a guardar edistribuir internamente como resultado da pesquisa, configurando-se em uma "evidência" dos resultados do processo de reorganização. A credibilidade do processo, principalmentejunto aos médicos - que costumam relutar em investir seu tempo nesse tipo iniciativa - dependia de uma argumentação baseada em evidências e na produção de informações sólidas ${ }^{7,15}$.

U mavez detectado econfirmado o queo hospital produzia (os procedimentos ou produtos), o quanto produzia e os insumos (materiais) necessários para produzir, estabeleciam-se padrões como o consumo médio mensal e as especificações para cada item.

A pesquisa de insumos também era ponto de partida para a criação de outros indicadores para acompanhar processos com relevância interdepartamental, como o nível de satisfação dos profissionais de saúde com a logística interna no diaa-dia. Assim, era importante obter feedback dos profissionais sobre a correspondência entre produto solicitado e distribuído, por exemplo. Ficava a cargo da Comissão Permanente de Padroni- zação de M aterial M édico-Hospitalar a elaboração e o acompanhamento desses indicadores.

0 aumento crescente da oferta de novos materiais e tecnologias em um cenário de restrição orçamentária e de grande pressão de forças de mercado vem impondo grandes desafios ao abastecimento, tanto no âmbito interno das organizações de saúde quanto no dos sistemas nacionais de saúde ${ }^{16}$. M ateriais mais complexos, cuja aquisição nos Estados Unidos eno Brasil tende a ser gerenciada pelos próprios médicos, podem responder por até $40 \%$ dos gastos com materiais. Portanto, a incorporação de novos materiais etecnologias precisava ser objeto de uma política clara de teste e validação pelo corpo clínico egerencial, tornando importante propor, ao longo do tempo, a criação de protocolos para validar os materiais incorporados. Diferentes materiais podem exigir critérios, tempos e protocolos de incorporação distintos, que variam segundo as definições de prioridades de cada instituição.

$M$ ateriais novos poderiam, em um primeiro momento, ser fornecidos em caráter "experimental", precisando ser validados para justificar uma incorporação definitiva. Em alguns hospitais, acompanhar essas incorporações podia também ser atribuição da Comissão Permanente de Padronização de Material M édico-Hospitalar ${ }^{7}$. Já existem al gumas publicações que sugerem critérios e parâmetros para nortear as incorporações tecnológicastanto em nível deorganizações quanto de sistemas de saúde ${ }^{16,17}$.

\section{Segunda etapa}

A segunda etapa da reorganização do abastecimento compreendia um ordenamento dos processos de trabalho de guarda e distribuição de insumos médico-hospitalares, de modo a preparar esse subsistema para operar dentro do novo enfoque de gestão e logística a partir do sistema produtivo. Para efetuar esse reordenamento, eram aplicadas técnicas da qualidadepara organizar o almoxarifado e a disposição dos estoques e estabel eciam-se protocolos para recebimento, conferência e classificação de materiais. 0 diferencial introduzido nesta etapa era a maior sensibilidade a questões clínicas da equipe da área de guarda e distribuição, propiciada pela participação nos grupos de problematizaçãa ${ }^{18}$.

Esta etapa podia, eventualmente, incluir também um redesenho do subsistema de compras, com a elaboração de cadastros qualificados de fornecedores, a capacitação da equipe para a elaboração de editais e a introdução de modalidades 
de aquisição de materiais que propiciassem maior agilidade nas relações com fornecedores (registro de preços, pregão) ou economias de escala (compras conjuntas com outros hospitais).

\section{Terceira etapa}

A terceira etapa contemplava o redesenho de toda logística de distribuição interna de materiais a partir de processos automáticos de reposição, possibilitados pelo acompanhamento eavaliação do uso desses materiais em função da produção efetivamente realizada.

Idealmente, o redesenho da distribuição interna poderia estar apoiado em um sistema informatizado que permitisse a atualização on line dos estoques de materiais do almoxarifado, baseada em informações sobre procedimentos realizados lançados em prontuários eletrônicos dos pacientes. A pesquisa de insumos possibilitava a criação deum sistema deinformações significativo para o cuidado do paciente (procedimentos), que poderia oportunamente ser acoplado a sistemas de informações sobre insumos. Informar a realização de um procedimento de punção venosa no paciente, por exemplo, é uma informação clinicamente relevante, cujo registro é bem mais compacto do quea descrição individual dosinsumos utilizados no procedimento (um escalpe ou cateter intravenoso, uma compressa de gaze não estéril, uma bola de algodão, um equipo injetor lateral, uma luva de procedimentos, três centímetros de esparadrapo e dez mililitros de álcool iodado, por exemplo). Uma informação sobre procedimento realizado poderia - pelo estabelecimento das matrizes insumo-produto - ser traduzida em informação sobre materiais utilizados.

$\mathrm{Na}$ prática, hospitais de portes e sofisticação tecnológica distintos podem beneficiar-se de desenhos compatíveis com seu grau de informatização nesta etapa. Na média dos hospitais públicos, porém, a informatização ainda á bastante incipiente. Portanto, o desenho proposto para esse contexto foi o de distribuição automática por entrepostagem.

Essa estratégia visava desonerar a enfermagem envolvida nas atividades clínicas do controle direto dos seus subestoques e da necessidade de elaboração quase cotidiana de pedidos de reposição de materiais. Era criado um entreposto de distribuição por andar ou setor, cujo responsável passava a ser um funcionário específico, de preferência originalmente ligado a atividades clínicas.

Identificar esse tipo de funcionário tendia a não ser difícil, haja vista que muitos auxiliares de enfermagem, por exemplo, são readaptados para exercerem novas atividades. As funções básicas desse funcionário eram repor estoques de cada insumo até os índices definidos como ideais na etapa de pesquisa de insumos, supervisionar a compatibilidade entre produção e consumo e monitorar a freqüência e duração das descontinuidades de abastecimento por tipo de insumo. Eventuais desvios dos padrões esperados de relação entre procedimentos efetuados e consumo de materiais seriam explorados em reuniões da Comissão Permanente de Padronização de Material M édico-Hospitalar.

O utra função que também podia ser assumida pela entrepostagem seria a confecção de kits de procedimentos em invólucros com códigos de barra personalizados para os setores sob sua responsabilidade, o que agilizaria a seleção dos materiais empregados nos procedimentos pela equipe clínica efacilitaria o controle de uso dos materiais.

\section{Discussão}

0 que a metodologia apresentada oferece como forma de contemplar as especificidades das organizações de saúde? Sua forma atual define um conjunto de propostas com escopo intra-organizacional, compatíveis com a governabilidade de atores que estejam atuando nesse plano. Alguns trabalhos referem significativos impactos dessas iniciativas internas de racionalização da cadeia de suprimentos, que podem reduzir os custos do abastecimento em até $15 \%$.

Fundamentalmente, a interface situada entre os profissionais da clínica e o setor de abastecimento é el eita como interface intra-organizacional crítica para a organização do abastecimento. A seleção de materiais e a gestão de estoques, em quese definem osmateriais utilizados no hospital e formas de acompanhar níveis de consumo que permitem programar a aquisição e a distribuição dos mesmos, são el eitos processos críticos para a organização do sistema de abastecimento.

Gerenciar a interface crítica passa a ser uma preocupação central da função de planejamento e abre o caminho para a intervenção sobre os dois processos críticos. A literaturainternacional já identifica essegerenciamento como fundamental para implantar uma abordagem "estratégica" da gestão de materiais, em oposição à abordagem tradicional ${ }^{7,14}$. No modelo tradicional degestão de materiais, a liderança dos processos de 
abastecimento fica exclusivamentea cargo defuncionários administrativos.

\section{Gestão da interface crítica}

A primeira medida proposta - a constituição de grupos interdisciplinares de qualidade, com definição de responsáveis pela condução do processo de reorganização do abastecimento na unidade - visa transpor o gap de credibilidade entre o sistema de abastecimento e a área clínica ${ }^{14}$.

Esse gap de credibilidade tanto está ligado à imagem desfavorável que os setores ligados a compras têm adquirido no serviço público quanto às freqüentes irregularidades no abastecimento. Em função da dinâmica de execução orçamentária, é muito comum o sistema de abastecimento distribuir menos do que foi utilizado, 0 que leva o usuário a pedir mais do que precisa. Como o usuário pede a mais, o sistema distribui menos, criando-se um ciclo de desconfiança recíproca, denominado por Vecina $\&$ Reinhardt ${ }^{3}$ de ciclo acumulativo.

Adicionalmente, no modelo tradicional, 0 permanente trade off entreo melhor produto eo menor custo costuma pender para o último, numa equação legitimada pelo princípio consagrado nas licitações públicas.

A constituição degruposinterdisciplinares de qualidade para a gestão de materiais (com sua posterior evolução para uma Comissão Permanentede Padronização de M aterial M édico-H ospitalar) pretende ser um primeiro passo para transpor o gap de credibilidade, incorporando sensibilidade a questões da clínica aos funcionários do abastecimento e conhecimento da dinâmica do abastecimento ao corpo clínico.

\section{Seleção de materiais}

A pesar de o número de materiais usados nas organizações de saúde não ser especialmente impressionante comparativamente a alguns segmentos industriais, a quantidade de produtos diferentes que podem ser gerados a partir desses materiais transforma essa cadeia de suprimentos em uma das mais complexas conhecidas. Um mesmo insumo pode ser empregado em uma enorme diversidade de produtos, cujas demandas oscilam no tempo. A dificuldade de planejar adequadamente 0 abastecimento é um reflexo dessa dinâmica.

A segunda preocupação central da metodologia, portanto, consiste em estabelecer uma lógica de seleção de materiais a partir de protoco- los padronizados de insumos necessários para a realização de cada procedimento, que vincule os insumos aos procedimentos em que são empregados. 0 momento da seleção dos insumos é também o momento em que se definem os crité rios de reposição de estoques, com base no registro do tipo de procedimentos em que os insumos são utilizados e no levantamento da quantidade de procedimentos de cada tipo realizada.

0 estabelecimento de "protocolos" de materiais para cada tipo de procedimento não é uma prática estranha aos profissionais de saúde. Principalmente nas especialidades cirúrgicas, já está bem estabelecido o uso de um conjunto de materiais permanentes padronizados, contidos nas "bandejas de procedimentos". A indústria de materiais médico-hospitalares vem tentando implantar a mesma lógica de padronização para materiais de consumo por procedimento, organizando kits demateriais por procedimento, aparentemente sem muito sucesso. I sso se deve ao fato de os processos de trabal ho e insumos utilizados na realização de um mesmo procedimento serem diferentes entre hospitais, entre distintos setores de um mesmo hospital e, até, entre profissionais de um mesmo setor. 0 tipo de paciente atendido, características pessoais dos profissionais e um treinamento realizado em instituições ou épocas diferentes podem determinar formas diversas de executar um mesmo procedimento.

0 diferencial da metodologia proposta em relação à aquisição de kits de material disponibilizados pela indústria de materiais médicos é a customização desses kits aos distintos setores do hospital, estabelecendo-se com o corpo de profissionais de saúdeuma relação defato de cliente, em que cliente é definido como al guém com direito de escolha' ${ }^{19}$.

Diversos trabalhos destacam a importância da padronização de materiais, da consideração das preferências do profissional, da participação de profissionais desaúde na equipe de suprimentos e de valorização profissional da equipeligada à gestão de materiais ${ }^{7,18,20}$. 0 estágio atual de desenvolvimento da metodologia incorpora claramente esses conceitos, que são estratégicos nas atividades de saúde e contribuem para abrandar a grande dicotomia entreatividades clínicas egerenciais nas organizações hospitalares ${ }^{21}$.

Gestão da programação de volume de aquisição e distribuição

Os procedimentos habituais de programação da distribuição nas organizações públicas 
incluem: a) distribuição automática baseada em cotas pré-definidas, pactuadas periodicamente com os serviços a partir da "experiência" de níveis de consumo; b) reposição automática com base em levantamento do histórico de consumo, que pode estar fortemente enviesado pela freqüência de intervalos de desabastecimento; c) reposição a partir da solicitação da própria unidade produtora, baseada na "experiência" de consumo.

$\mathrm{Na}$ iniciativa privada, a contabilização dos gastos individuais dos pacientes com materiais é um dos componentes da elaboração das contas hospitalares e pode nortear a reposição dos estoques. Essa modalidade de gerenciamento dos estoques de materiais tem a desvantagem de induzir substanciais margens de erro. Os erros advêm do enorme trabalho de escrituração envolvido no rastreamento dos insumos individualmente utilizados em cada paciente e, principalmente, do fato de a informação sobre insumos ter pouca relevância para o cuidado do paciente, ao contrário do que se verifica quando a unidade de registro do consumo são os procedimentos clínicos e cirúrgicos realizados pelos profissionais de saúde.

A metodologia apresentada propõe-se a transformar a forma de programação dominante de aquisição e distribuição de insumos em hospitais públicos, baseada na "experiência", em distribuição baseada em "evidência", a partir do estabelecimento de uma correlação entre insumos e produtos. A lógica assim estabelecida, que pode ser exportada para um sistema informatizado de gestão do abastecimento, acompanha as tendências da evolução dos sistemas de informação em organizações de saúde. Desde o início deste século, vem sendo observada na saúde uma tendência a aumentos de investimento em sistemas deinformações clínicas e de redução na aquisição de sistemas informatizados do tipo ERP (enterprise resource planning), centrados no gerenciamento de recursos materiais organizacionais. As "matrizes insumos-produtos" para programação do abastecimento permitem uma "tradução" desta segunda lógica para a primeira ${ }^{22}$.

Outra possibilidade interessante é a de utilizar os procedimentos assim estruturados para desenhar um sistema de custos baseado em atividades, que poderia contribuir para a determinação de custos e facilitar o trabalho de auditorias de contas hospitalares.

\section{Correlações \\ com referenciais teóricos da área}

A gestão de materiais em organizações de saúde é abordada na literatura segundo duas perspectivas: na perspectiva endógena do abastecimento ou segundo uma visão mais ampla, sugerida pela filosofia da qualidade e pela gestão da cadeia de suprimentos.

$\mathrm{N}$ a perspectiva mais endógena da função de gestão de materiais, parte-se de uma individualização relativamente estanque do abastecimento em setores que executariam as funções de sel eção e padronização de insumos, guarda e distribuição de materiais, controle de estoques e compras. A prioridade nas iniciativas de organização do abastecimento recai sobre a organização dos processos internos de cada um dos setores ${ }^{3,5}$.

As visões mais abrangentes encaram a função de gestão do abastecimento de materiais como uma das funções integrantes das cadeias de suprimentos e de valor da organização ${ }^{7}$. A literatura clássica que associa qualidade à gestão da cadeia de suprimentos aborda temas que tradicionalmente são separados por acadêmicos da engenharia de produção e pelos profissionais de serviços em duas áreas: (a) coordenação intraorganizacional (ou gerenciamento tradicional da qualidade), na perspectiva interna da cadeia de suprimento; (b) integração inter-organizacional, que estuda a interface entre cadeia de su primentos e metodologias de qualidade a partir de um foco externo da cadeia de suprimentos ${ }^{15}$.

Em termos conceituais, a metodologia apresentada nasce atrelada à filosofia da qualidade. D efine os profissionais de saúde como "clientes" do sistema de abastecimento e endossa o respeito ao "foco no cliente" recomendado pela qualidade, tendo assim avançado para reconhecer a necessidade de envolver toda a organização no planejamento do abastecimento.

$\mathrm{N}$ a perspectiva de gerenciamento da cadeia de suprimentos como integração inter-organizacional, passa a ser relevante realizar uma análise integrada de toda a cadeia produtiva, desde o desenvolvimento do produto até as formas de aumentar a integração com fornecedores e consumidores. Os princípios da qualidadetotal, definidos por Juran e D eming ${ }^{23}$ - abordagem sistêmica, agregação de valor para o cliente, decisões baseadas em fatos e melhoria contínua - podem ser utilizados para desenhar e operar processos que garantem o bom desempenho da cadeia desuprimentos em extensões cada vez mais amplas ${ }^{24}$. 


\section{Conclusões}

A metodologia proposta apresenta um sistema de organização da programação de insumosque pode importar em avanços significativos para a organização do abastecimento hospitalar. É baseada em dados pertinentes para o cuidado do paciente (procedimentos em oposição a insumos) e sustentada pela criação de vínculos institucionais entre distintos departamentos da organização ${ }^{22}$. Em que pesem as ressalvas à aplicação de ferramentas da qualidade em organizações de saúde ${ }^{19}$, os princípios da qualidade parecem ter utilidade no contexto estudado.

As etapas já desenvolvidas da metodologia conformam ainda uma abordagem com um foco predominantemente intra-organizacional e centrado no produto. Entretanto, as formulações teóricas da gestão de cadeia de suprimentos absorvem premissas da gestão pela qualidadeeavançam no sentido de considerar que a qualidade do produto é apenas um dos aspectos de programas demelhoria contínua filiados à qualidade ${ }^{24}$. A "satisfação do cliente final" só é possível se toda a cadeia de suprimentos estiver comprometida e integrada em práticas coerentes e eficazes.

Com isso, as tendências mais modernas das iniciativas da qualidade na cadeia de suprimentos deixam de ter um foco exclusivamente intra- organizacional para tentar promover a interação e sincronia ao longo de toda a rede de organizações, processos e pessoas que integram uma cadeia de suprimentos.

Nessa perspectiva, a metodologia apresentada caminha no sentido de se acompanhar de outros desenvolvimentos. Esses desenvolvimentos incluem tanto aprimorar processos que envolvam parceiros externos - na formação de grupos de compradores ${ }^{25}$ e nas formas de relacionamento e contrato com fornecedores ${ }^{15}$ - quanto na persistência do compromisso com a melhoria contínua dos processos internos à organizaçãa $0^{5}$ e com uma crescente incorporação da perspectiva do paciente.

No contato com os serviços de saúde, o paciente não busca o consumo de produtos (procedimentos), busca o desfecho ou resultado advindo desse consumo ${ }^{26}$. 0 desfecho esperado do consumo de um produto ou conjunto de produtos seria a melhora do estado de saúde do paciente. A seleção de produtos a ser oferecida ao paciente, no entanto, é definida pelo profissional de saúde. Comprometer o profissional com uma visão crítica e responsável sobrea necessidade ea melhor seleção dos produtos, na perspectiva do resultado sobre a saúde do paciente, é sempre um objetivo central de ações da qualidade em organizações de saúde. 


\section{Colaboradores}

M Infante participou do desenvolvimento da metodologia, do trabal ho de campo, da concepção teórica do artigo e da revisão final. M A Borges dos Santos participou da concepção teórica do artigo, da revisão bibliográfica e da redação do texto.

\section{Referências}

1. Neil R The ol' switcheroo. Using knowledge from other industries. $\mathrm{M}$ ater $\mathrm{M}$ anage $\mathrm{H}$ ealth Care 2004; 13 (5): $14-8$.

2. Lambert DL, Cooper M. Issues in Supply Chain $M$ anagement. Indust M arket $M$ anage 2000; 29:65-83.

3. Vecina Neto G, Reinhardt Filho W. Gestão de recursos materiais e de medicamentos. Série Saúde e Cidadania, vol. 12. São Paulo: Faculdade de Saúde Pública da Universidade de São Paulo; 2002.

4. Healthcare Financial $M$ anagement Organization. HFM A's 2005 Supply Chain Benchmarking Survey. [acessado 2005 Set 05]. Disponível em: www.hfma. org/resource/mckeeson benchmark2005.pdf.

5. Dacosta-Claro I, Lapierre SD. Benchmarking as a tool for the improvement of health services supply departments. Health Serv M anage Res 2003; 16:213223

6. Davis RN. No more chances for supply-chain savings? Look again! H ealthc Financ M anage 2004; 58(1): 68-75.

7. Ballard R. Strategic supply cost management: physician preference without deference. Healthc Financ $M$ anage 2005; 59(4):78-80, 82, 84.

8. Sommer R, Amick T. Pesquisa-ação: Ligando pesquisa a mudança organizacional. Série Planejamento de Pesquisas nas Ciências Sociais, no 4. Brasília: UnB/ Laboratório de Psicologia Ambiental; 2003.

9. Coughlam P, Coghlam D. Action research for operations management. Int J O per Prod M an 2002; 22 (2): 220-240.

10. Westbrook R Action research: a new paradigm for research in production and operations management. Int J Oper Prod Man 1995; 15(12): 6-20.

11. Health Manpower Development Staff. District and $\mathrm{National}$ Planning and M anagement Workshop Manual. The MEDEX Primary Health Care Series. Honolulu, Hawaii: John A. Burns School of Medicine/ University of Hawaii; 1983.

12. Brassard M. Qualidade: ferramentas para uma melhoria contínua. Rio de Janeiro: Editora Qualitymark; 1992.
13. Nogueira RP. Perspectivas da qualidade em saúde. Rio de Janeiro: Editora Qualitymark;1994.

14. Robinson CJ, Malhotra MK. Defining the concept of supply chain quality management and its relevance to academic and industrial practice. Int J Prod Econ 2005; 96 (2005):315-337.

15. Neumann L. Streamlining the supply chain. Healthc Financ $M$ anage 2003; 57(7):56-62.

16. Johnson-M asoti AP, Eva, K. Decision-M aking Framework for the Priorization of Health Technologies. [acessado 2005 Abr 20]. Disponível em: http:// jdi.econ.queensu.ca/Files/Conferences/H ealthServicesconferencepapers/Johnson-M asotti_paper.pdf

17. National Institute for Clinical Excellence (NICE). Guide to the Methods of Technology Appraisal (reference N 0515); 2004.

18. Dejohn P. Politics of preference back in style at AHRM M. H osp M ater M anage 2004; 29(9):1,10-2.

19. Gurgel Junior GD, Vieira MMF. Qualidade total e administração hospitalar: explorando disjunções conceituais. Rev C S Col 2002; 7(2):325-334.

20. Hesson D. Indiana system finds standardizing pays off. Hosp M ater M anag 2004; 29(8):2, 10-1.

21. Cecílio LCO. A modernização gerencial dos hospitais públicos. 0 difícil exercício da mudança. Rev Adm Publica 1997; 31(3):36-47.

22. Solovy A. Health care's most wired. A wired exchange. Mater M anag Health Care 2004; 13(8):27-31.

23. Hackman JR, Wageman R. Total quality management: empirical, conceptual and practical issues. Admin Sci Quart 1995; 40(2):309-342.

24. Lee HL, Wang S. Higher supply chain quality with lower cost: Lessons from total quality management. Int. J. Prod Econ 2005; 96:289-300.

25. Parker R. Purchasing and supply: Scope out the competition. Health Serv J 2003; 113 (5580):13-14.

26. Grossman M On the concept of health capital and the demand for health. J Polit Econ 1972; 80(2):223255.

Artigo apresentado em 19/05/2006

Artigo aprovado em 07/02/2007

Versão final apresentada em 04/04/2007 